\title{
TOPOLOGICAL CLASSIFICATION OF GENERIC REAL MEROMORPHIC FUNCTIONS FROM COMPACT SURFACES
}

\author{
Antonio F. Costa, Sergey Natanzon and Boris Shapiro \\ UNED, Facultad de Ciencias, Departamento de Matemáticas Fundamentales \\ C. Senda del rey, 9, 28040 Madrid, Spain; acosta@mat.uned.es \\ National Research University Higher School of Economics (HSE) \\ 20 Myasnitskaya ulitsa, Moscow 101000, Russia; natanzons@mail.ru \\ Stockholm University, Department of Mathematics \\ SE-106 91 Stockholm, Sweden; shapiro@math.su.se
}

\begin{abstract}
In this article, to each generic real meromorphic function (i.e., having only simple branch points in the appropriate sense) we associate a certain combinatorial gadget which we call the park of a function. We show that the park determines the topological type of the generic real meromorphic function and the set of parks produce a stratification of the space of generic real meromorphic functions. For any of the above topological types, we introduce and calculate the corresponding Hurwitz number. Finally we relate the topological types of generic real meromorphic functions with the monodromy of orbifold coverings.
\end{abstract}

\section{Introduction}

The main object of study in the present paper is the Hurwitz space $\mathbf{R} H$ of real meromorphic functions on compact surfaces. Its structure is substantially more complicated than that of the classical Hurwitz space $H$ of complex meromorphic functions. The latter space consists of pairs $(P, f)$ where $P$ is a complex algebraic curve, i.e., a compact orientable Riemann surface and $f: P \rightarrow \overline{\mathbf{C}}$ is a meromorphic function, i.e., a holomorphic map to the Riemann sphere. Each point $(P, f)$ may be described by polynomial equations with complex coefficients providing a natural topology to the space $H$.

In applications one often encounters generic meromorphic functions, i.e., functions forming a dense subset $H^{0} \subset H$ which is stable with respect to arbitrary small perturbations of functions. For complex meromorphic functions, $H^{0}$ consists of functions with all simple critical values. In other words, $H^{0}$ contains all functions $f$ whose branchings are of second order and such that the images of all branchings under $f$ are pairwise distinct, i.e., the critical values of $f$ are simple.

According to the basic classical results of Hurwitz [6], the space $H$ may be stratified and each stratum consists of all functions of a given degree $d$ on curves of a given genus $g$. (Such stratum is denoted by $H_{g, d}$.) Analogously we can stratify $H^{0}$ by strata containing generic complex functions of degree $d$ on curves of genus $g$. (Each stratum is denoted by $H_{g, d}^{0}$.) Obviously, $H_{g, d}^{0} \subset H_{g, d}$ and one can show that the difference $H_{g, d} \backslash H_{g, d}^{0}$ has real codimension two in $H_{g, d}$ which explains why strata of $H$ are in $1-1$-correspondence with that of $H^{0}$.

https://doi.org/10.5186/aasfm.2018.4319

2010 Mathematics Subject Classification: Primary 14P25; Secondary 14E22, 37F10.

Key words: Real meromorphic functions, gardens and parks, Hurwitz numbers. 
Critical values of functions from $H_{g, d}^{0}$ provide the complex structure on $H_{g, d}^{0}$ (and on $H_{g, d}$ as well) induced by $H$. The number of functions belonging to $H_{g, d}^{0}$ with a given set of $2 d+2 g-2$ distinct critical values was defined and in many cases calculated by Hurwitz in [6]. (Observe that $2 d+2 g-2$ is also the dimension of $H_{g, d}^{0}$ over C.) The latter number of functions is classically referred to as the corresponding Hurwitz number. Hurwitz numbers play important role in modern algebraic geometry and mathematical physics.

The space $H$ is endowed with a natural involution $\tau_{H}: H \rightarrow H$ anti-holomorphic with respect to the complex structure on $H$. This involution associates to a pair $(P, f)$ the pair $(\bar{P}, \bar{f})$, where the Riemann surface $\bar{P}$ is obtained from $P$ by substituting all local charts on $P$ by their complex-conjugates and by using $\bar{f}(z)=\overline{f(z)}$. The set of fixed points of $\tau_{H}$ coincides with the space $\mathbf{R} H$ of all real meromorphic functions.

Let us now discuss the structure of $\mathbf{R} H$. By definition, for $(P, f) \in \mathbf{R} H$, one has that $\tau_{H}(P, f)=(P, f)$. Therefore, $\tau_{H}$ generates an anti-holomorphic involution $\tau: P \rightarrow P$ such that $f(\tau(p))=\overline{f(p)}$. The category of such pairs $(P, \tau)$ is isomorphic to the category of real algebraic curves, see [1]. Under this isomorphism, real algebraic functions correspond to morphisms of real algebraic curves to $(\overline{\mathbf{C}}$, con $j)$, where $\operatorname{conj}(z)=\bar{z}$.

The set of strata of $\mathbf{R} H$ is substantially more complicated than that of $H$, see $[4,3,9,10,11,8]$. (We will discuss these strata in details in $\S 5$.) As in the complex case, the most important real meromorphic functions for applications are generic ones; these functions are the main object of study here.

As in the complex case, they form an open, dense and stable with respect to arbitrary small deformations subset $\mathbf{R} H^{0} \subset \mathbf{R} H$. But in the real situation the definition of $\mathbf{R} H^{0}$ becomes more involved. In particular, $\mathbf{R} H^{0}$ does not coincide with $\mathbf{R} H \cap H^{0}$ since the closure of the latter intersection is strictly smaller than $\mathbf{R} H$. To improve the situation, one has to define $\mathbf{R} H^{0}$ as the set of all real meromorphic functions whose branchings are of the second order and for the images of these branchings, i.e., for the critical values, one should require that

(i) critical values are distinct when they are non-real;

(ii) critical values are the images of at most two branchings when they are real.

In the latter case, when there are two branchings mapped to the same real critical value, they must necessarily be interchanged by the involution $\tau$ on $P$. Therefore, the set $\mathbf{R} H^{0}$ defined in the above way is stable under arbitrary small deformations inside the class of real meromorphic functions. On the other hand, $\mathbf{R} H^{0}$ is dense in $\mathbf{R} H$. Indeed, for any given real meromorphic function, using small perturbations symmetric with respect to $\tau$, one can achieve that all branchings of its small perturbations will be of order two and, therefore, belong to $\mathbf{R} H^{0}$.

Contrary to the complex case, there are many more strata of the space $\mathbf{R} H^{0}$ than in the space $\mathbf{R} H$ which, in the first place, is related to the fact that $\mathbf{R} H \backslash \mathbf{R} H^{0}$ has real codimension one. To describe the topological invariants distinguishing strata of $\mathbf{R} H^{0}$, we have to introduce a rather complicated combinatorial gadget which we call a park of a real meromorphic function. This notion is similar to the notion of a garden introduced in our earlier paper [13] and which describes strata in the space $\mathbf{R} H \cap H_{0, d}^{0}$ of real generic rational functions. (The notion of a park is substantially more complicated.) In [12] there is a classification of a particular class of generic real meromorphic functions and now we shall present the complete classification of these functions. 
The structure of this paper is as follows. In $\S 2$ we define a park which, as we will see later, uniquely determines the topological type of generic real meromorphic functions. In $\S 3$ we explain how to associate a park to an arbitrary generic real meromorphic function. In $\S 4$ we show that given an arbitrary abstract park $\mathcal{P}$, we can always find a generic real meromorphic function whose associated park coincides with $\mathcal{P}$. Let $H(\mathcal{P})$ denote the space of functions corresponding to a given park $\mathcal{P}$. We show that $H(\mathcal{P})$ is connected. In $\S 5$ we recall some basic information about the classification of real meromorphic functions, see $[9,10,11]$ and explain how to identify the strata of the space $\mathbf{R} H$ coinciding with the sets $H(\mathcal{P})$. In $\S 6$ we define and calculate the corresponding Hurwitz number for $H(\mathcal{P})$. Finally, in $\S 7$ we explain how parks are related to the monodromy of coverings of orbifolds.

Acknowledgements. The article was prepared within the framework of the Academic Fund Program at the National Research University Higher School of Economics (HSE) in 2017-2018 (grant 17-01-0030) and by the Russian Academic Excellence Project "5-100". The first author was supported by the Spanish Ministry Project MTM2014-55812-P while the third author wants to acknowledge the hospitality and the financial support of the Higher School of Economics during his visit to Moscow in Spring 2014.

\section{Gardens and parks}

In order to motivate the main definition we shall start with an example.

Example 1. Let $f: P \rightarrow \overline{\mathbf{C}}$ be a generic real meromorphic function from a Riemann surface $P$ of genus 8 such that $f$ has two real critical values. Now we construct a combinatorial object that will determine the topological type of $(P, f)$.

Consider in $\overline{\mathbf{C}}$ the hemispheres $\mathbf{C}_{+}$and $\mathbf{C}_{-}$of complex numbers with positive and negative imaginary part respectively. Let $U$ be a symmetric annular neighbourhood of the real cycle $\overline{\mathbf{R}}$ which contains only real critical values. Its boundary $\partial U$ consists of the contours $\mathcal{C}_{+} \subset \mathbf{C}_{+}$and $\mathcal{C}_{-} \subset \mathbf{C}_{-}$. We shall denote by $D_{+} \subset \mathbf{C}_{+}$and $D_{-} \subset \mathbf{C}_{-}$ the two connected components of $\overline{\mathbf{C}} \backslash U$.

We consider the set $S=f^{-1}\left(\mathcal{C}_{+}\right) \cup f^{-1}\left(\mathcal{C}_{-}\right)$of simple contours in $P$. We cut the surface $P$ by the contours in $S$, we assume that in our example the result is:

- a sphere with four holes $P_{R}=f^{-1}(U)$ ( $f$ restricted to $P_{R}$ is a two-fold covering of the annulus $U$ ) and

- two (possibly disconnected) surfaces with boundary: $P^{+}=f^{-1}\left(D_{+}\right)$and $P^{-}=f^{-1}\left(D_{-}\right)$.

The covering $f: P_{R} \rightarrow U$ can be extended to a real rational meromorphic function $g$ which is described by an object called garden. In this case the garden is given by the preimage by $g$ of $\overline{\mathbf{R}}$, decomposing $\overline{\mathbf{C}}$ in four regions and a bicolouration of the four regions (white for the preimage of $\mathbf{C}_{+}$and black for the preimage of $\mathbf{C}_{-}$.) In other cases there are other elements but they are not essential in our example: the enumeration of the preimage of real critical values and some numbers related to the degree of $f$ on the preimage of real points.

Our results will imply that the topological type of $(P, f)$ depends on the number of connected components of $P^{+}$and $P^{-}$(the number of entrances and exits), the genera of the components of $P^{+}$and $P^{-}$(weights of entrances and exits), the way of connecting the boundaries of $P^{+}$and $P^{-}$with the boundaries of $P_{R}$ (encoded by the alleys connecting the white regions with entrances and the black regions with 
exits.) In our example, we have one entrance and one exit of genus three that must be joined by alleys as shown in the Figure 2 .

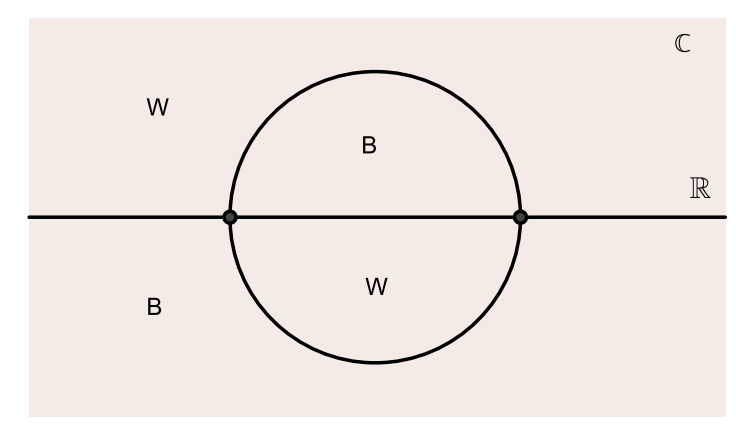

Figure 1. The garden of Example 1. The bicolouration of the faces is marked using the letters $\mathrm{W}$ and $\mathrm{B}$.

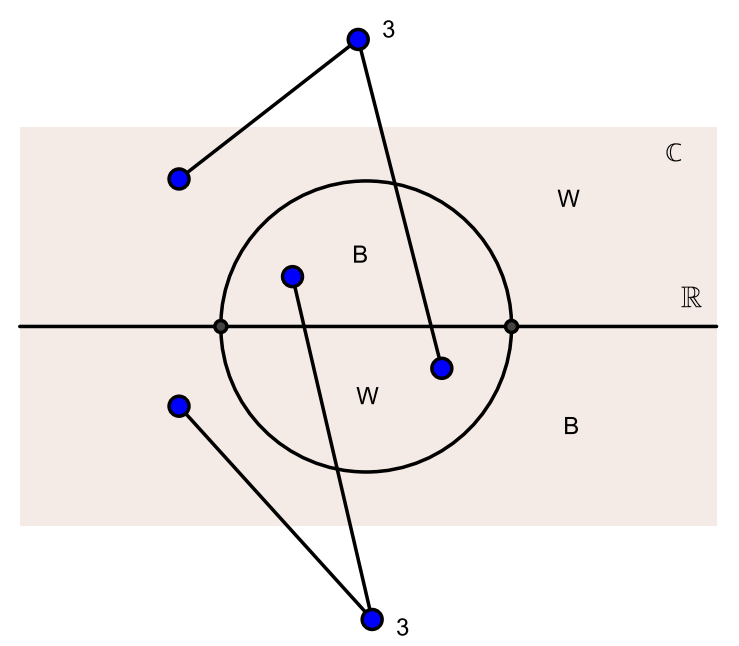

Figure 2. The park of Example 1.

To define our main combinatorial object: park, we need four preliminary concepts: orientable garden, oriented separated garden, non-orientable garden and nonoriented separated garden.

2.1. Orientable gardens. A semi-garden $S \mathcal{G}$ is a structure composed of an oriented surface, chords, simple contours, faces, edges, a colouration of faces, lengths of edges and a cyclic order of the vertices. Now we describe each one of such ingredients. The first one is a compact connected oriented differentiable surface $P$ with or without boundary together with a collection of chords, i.e., non-selfintersecting curves connecting points on the boundary of $P$ and simple contours, i.e., closed nonselfintersecting curves embedded in $P$. Connected components of the boundary of $P$, the chords and the contours of $S \mathcal{G}$ may intersect transversely, but can not be tangent to each other. The set of chords and simple contours splits $P$ into simply-connected domains called faces. Intersection points of chords and contours together with the endpoints of chords are called vertices. Vertices split the boundary of each face into curve segments or closed curves called edges. One additionally requires that the end points of chords are pairwise distinct and that any vertex which is an intersection point of chords and/or contours lies in the closure of exactly four edges. 
A semi-garden $S \mathcal{G}$ is additionally equipped with

(i) black and white colouring of the faces such that every two neighbouring faces have different colours;

(ii) positive integers ("lengths") assigned to its edges;

(iii) a cyclic order on the set of all vertices.

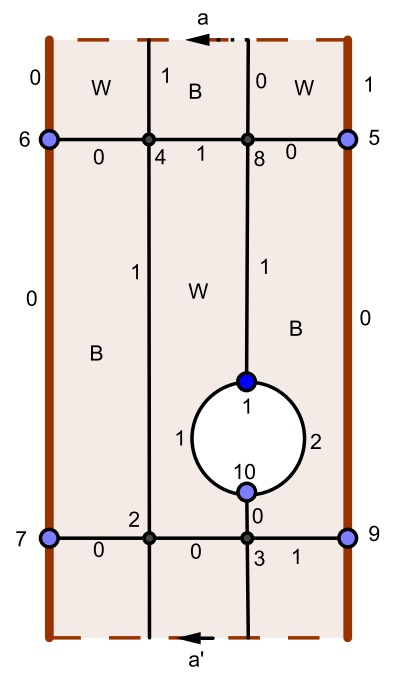

Figure 3. A semi-garden: $P$ has genus 0 and three boundary components. The sides $a$ and $a^{\prime}$ must be identified.

Let $P^{\prime}$ be the surface $P$ with the opposite orientation. For a given semi-garden $S \mathcal{G}$, the semi-garden obtained by considering the surface $P^{\prime}$, the chords, simple contours, lengths of edges and cyclic order of the edges of $S \mathcal{G}$, but interchanging black and white colours of faces, is called the conjugate semi-garden $\overline{S \mathcal{G}}$ of $S \mathcal{G}$.
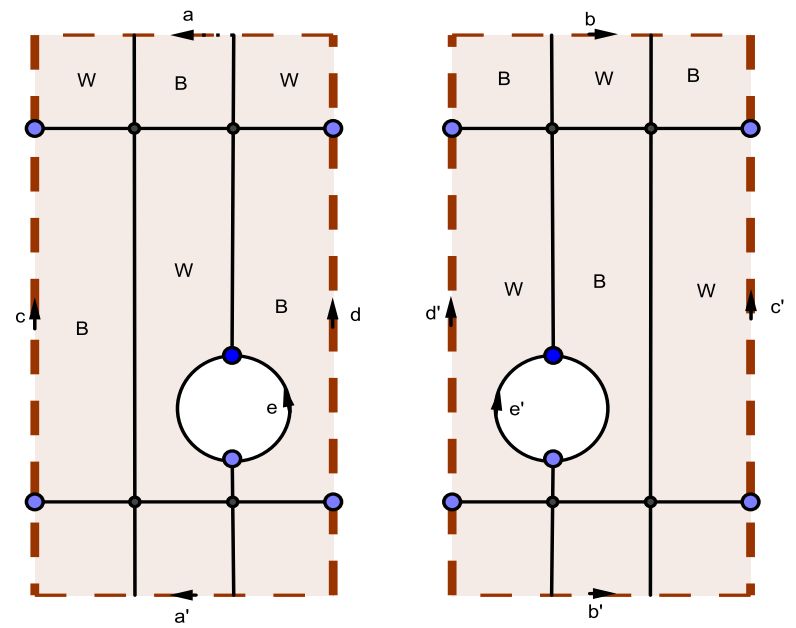

Figure 4. An orientable garden. To obtain $\widetilde{P}$ it is necessary to identify $a$ with $a^{\prime}, b$ with $b^{\prime}, c$ with $c^{\prime}$ and $e$ with $e^{\prime}$, producing a surface of genus 2 .

By an orientable garden we call a pair of conjugate semi-gardens $S \mathcal{G}$ and $\overline{S \mathcal{G}}$ defined on surfaces with non-empty boundary. To an orientable garden we associate a Riemann surface $\widetilde{P}$ obtained in the following way. Let $P$ and $P^{\prime}$ be the surfaces corresponding to $S \mathcal{G}$ and $\overline{S \mathcal{G}}$ respectively, we consider $P$ and $P^{\prime}$ as disjoint surfaces with an orientation reversing diffeomorphism $h: P \rightarrow P^{\prime}$ sending the elements of the orientable garden $S \mathcal{G}$ to the elements of $\overline{S \mathcal{G}}$ (the diffeomorphism $h$ sends black faces to white faces) and we identify the points of the boundaries of $P$ with the ones of $P^{\prime}$ 
by the map $h$. The surface $\widetilde{P}$ has an orientation-reversing involution $\nu$ (given by $h$ and $h^{-1}$ ) sending the initial semi-garden to its conjugate. The quotient surface $\widetilde{P} / \nu$ is an orientable surface isomorphic to $P$. Thus an orientable garden is given by a compact orientable surface $\widetilde{P}$ without boundary with an anticonformal involution $\nu$ such that $\widetilde{P} / \nu$ is orientable and an additional structure provided by a semi-garden on $\widetilde{P} / \nu$.

Figure 4 shows an orientable garden associated to a genus 2 surface; the corresponding semigardens are the one represented in Figure 3 and its conjugate.

2.2. Oriented separated garden. An elementary orientable garden is given by the Riemann sphere with the standard complex conjugation $\sigma$ and a given integer "length" of the real cycle (the corresponding semi-gardens have neither chords nor simple contours.) Now consider a pair of elementary orientable gardens $\left\{\left(\mathcal{G}_{i}, \sigma_{i}\right): i=\right.$ $0,1\}$ with equal lengths of their real cycles. Take the identical isomorphisms $\phi_{i}$ : $\left(\mathcal{G}_{i}, \sigma_{i}\right) \rightarrow\left(\mathcal{G}_{1-i}, \sigma_{1-i}\right)$ and the involution $\sigma:=\phi_{i} \sigma_{i}$ of $\mathcal{G}_{0} \cup \mathcal{G}_{1}$. Call the obtained structure $\left(\mathcal{G}_{0} \cup \mathcal{G}_{1}, \sigma\right)$ an oriented separated garden.

2.3. Non-orientable gardens. A symmetric semi-garden $S S \mathcal{G}$ is a pair consisting of a semi-garden on a surface $P$ without cyclic enumeration of its vertices and an orientation-reversing involution $\tau: P \rightarrow P$ without fixed points which changes the colour of the faces and preserves the "lengths" of the edges. In a symmetric semigarden $S S \mathcal{G}$ the cyclic enumeration of the vertices of a semi-garden is substituted by the cyclic numeration of pairs of vertices which are interchanged by the involution $\tau$.

A non-orientable garden is a symmetric semi-garden $S S \mathcal{G}$ and an associated orientable surface $\widetilde{P}$ obtained identifying all pairs of points on the boundary of $P$ interchanged by $\tau$. The involution $\tau$ induces an orientation-reversing involution $\widetilde{\tau}$ on $\widetilde{P} \cdot \operatorname{Fix}(\widetilde{\tau})$ does not separate $\widetilde{P}$, then $\widetilde{P} / \widetilde{\tau}$ is non-orientable.

Thus a non-orientable garden is given by a compact connected orientable surface $\widetilde{P}$ without boundary, an orientation reversing involution $\widetilde{\tau}$ such that $\widetilde{P} / \widetilde{\tau}$ is nonorientable and with a symmetric semi-garden structure on $\overline{\widetilde{P}-\operatorname{Fix}(\widetilde{\tau})}$. Note that $\operatorname{Fix}(\widetilde{\tau})$ may be empty and in this case $P$ has no boundary.

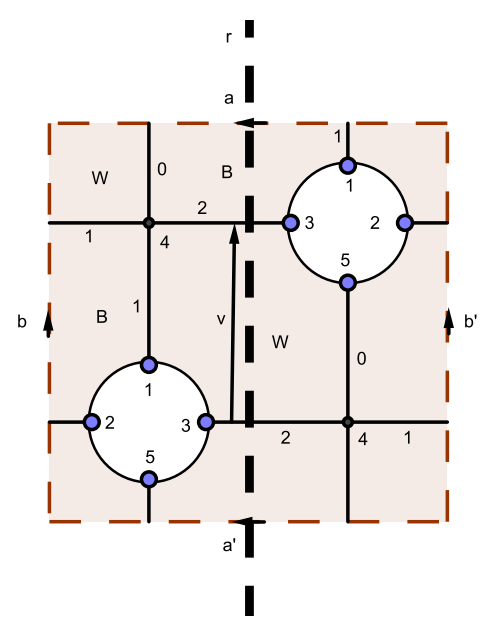

Figure 5. A symmetric semi-garden. The sides $a$ and $a /, b$ and $b /$ must be identified to obtain a torus with two boundary components.

The Figure 5 shows a symmetric semi-garden corresponding to a surface $P$ of genus one with two boundary components. The orientation-reversing involution of $P$ is given by the glide-reflection of the axis $r$ and vector $v$. The non-orientable garden 
given by the symmetric semi-garden is associated to a surface of genus two and the orientation-reversing involution has one contour as fixed point set (one oval).

2.4. Non-oriented separated garden. A elementary non-orientable garden is the Riemann sphere with the antipodal involution $\tau: z \rightarrow-\bar{z}^{-1}$, a given integer "length" of the real cycle, and a given colouring of the hemispheres into which the real cycle splits the Riemann sphere. Consider a pair of elementary non-orientable gardens $\left\{\left(\mathcal{G}_{i}, \tau_{i}\right) \mid i=0,1\right\}$ with equal lengths of their real cycles. Take the identical isomorphisms $\phi_{i}:\left(\mathcal{G}_{i}, \sigma_{i}\right) \rightarrow\left(\mathcal{G}_{1-i}, \sigma_{1-i}\right)$ and the involution $\sigma:=\phi_{i} \tau_{i}$ of $\mathcal{G}_{0} \cup \mathcal{G}_{1}$. The obtained structure $\left(\mathcal{G}_{0} \cup \mathcal{G}_{1}, \sigma\right)$ is called a non-oriented separated garden.

A separated garden is either an oriented or non-oriented separated garden.

2.5. Parks. A park is a structure consisting of

(i) (orientable and non-orientable) gardens and (oriented and non-oriented) separated gardens;

(ii) entrances and exits;

(iii) alleys,

which we define below.

An entrance (resp. exit) is a point coloured white (resp. black) and endowed with a non-negative integer called weight. By an alley we call a path connecting an entrance or an exit with a face of a garden or a separated garden.

The elements of a park satisfy the following:

(i) alleys connect entrances to white faces and exits to black faces;

(ii) each face has exactly one alley;

(iii) at least one alley is connected to each entrance and each exit;

(iv) there exists an involution sending entrances to exits (preserving weights), alleys to themselves and acting on gardens and separated gardens as their associated involutions.

Note that the information on the number of exits, alleys from exits and its weights may be deduced from the corresponding concepts on entrances and vice-versa. We maintain the two types of elements for aesthetical reasons.

Two parks are called isomorphic if there exist homeomorphisms of the associated surfaces to the gardens and bijections between the sets of entrances and exits preserving all elements and relations of the structures.

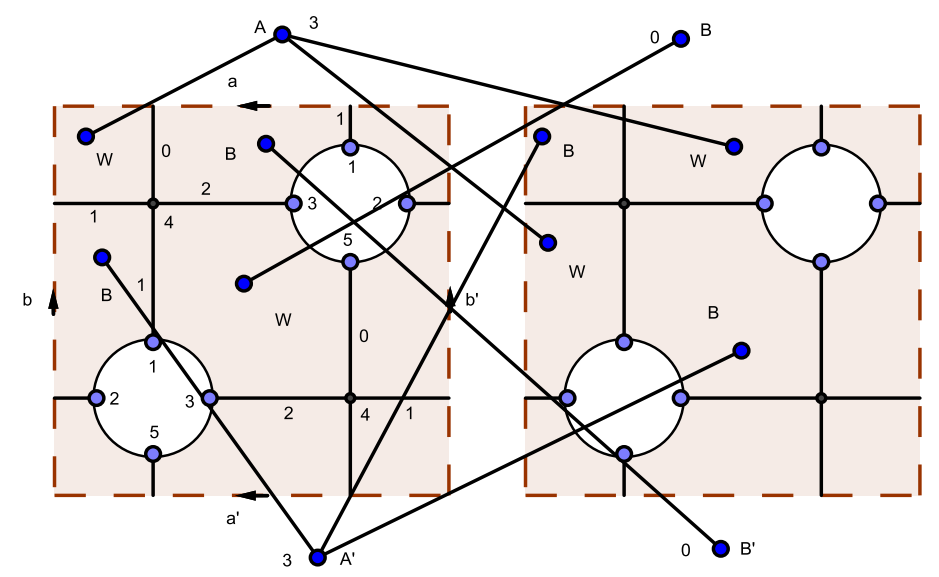

Figure 6. Example of a park. 
The Figure 6 shows a park constructed from two non-orientable gardens as the one in Figure 5. In the Example 3 of the next section we describe the generic real meromorphic functions with the topological type represented by this park.

\section{Park corresponding to a real meromorphic function}

Recall that a real algebraic curve is a pair $(P, \tau)$ consisting of a compact Riemann surface $P$ and an anti-holomorphic involution $\tau: P \rightarrow P$. A real meromorphic function on $(P, \tau)$ is a holomorphic function $f: P \rightarrow \overline{\mathbf{C}}$ such that for any $p \in P$, $f(\tau(p))=\overline{f(p)}$. A real meromorphic function $f$ is called generic if (i) all critical points $p \in P$ have degree 2 , and (ii) if critical points $p_{1} \neq p_{2} \in P$ have the same image $z=f\left(p_{1}\right)=f\left(p_{2}\right)$, then $z \in \overline{\mathbf{R}}$ and $\tau\left(p_{1}\right)=p_{2}$.

Let us now associate a park to any generic real meromorphic function.

3.1. Associated gardens. The real cycle $\overline{\mathbf{R}} \subset \overline{\mathbf{C}}$ splits the Riemann sphere into the hemispheres $\mathbf{C}_{+}$and $\mathbf{C}_{-}$with positive and negative imaginary part respectively. We will call them resp. the white and the black hemispheres.

Consider a symmetric neighbourhood of a real cycle $U \supset \overline{\mathbf{R}}$ (i.e., $\bar{U}=U$ ) which contains only real critical values. Its boundary $\partial U$ consists of the contours $\mathcal{C}_{+} \subset \mathbf{C}_{+}$ and $\mathcal{C}_{-} \subset \mathbf{C}_{-}$.

Take the preimage $f^{-1}(U)$. Contracting each connected component of the boundary of the preimage to a point, we obtain a finite family $\Phi$ of compact connected Riemann surfaces. The preimage $f^{-1}(\overline{\mathbf{R}})$ splits each of them into simply-connected domains. The closures of these domains are called faces. Each face contains exactly one point obtained as the result of the contraction of a component in $f^{-1}(\partial U)$. Let us colour the face white if this point is obtained by contracting of the inverse image of $f^{-1}\left(\mathcal{C}_{+}\right)$and black if it is obtained by contracting $f^{-1}\left(\mathcal{C}_{-}\right)$.

Contracting each of the contours $\mathcal{C}_{ \pm}$to a point, we get a sphere $\bar{U}$ with two labelled points $z_{ \pm}$. The real cycle splits it into the white and the black hemispheres. Function $f$ induces the branched covering $\bar{f}$ of $\bar{U}$ by the family $\Phi$ which maps white faces to the white hemisphere and black faces to the black one. Points $z_{ \pm}$will be the only non-real critical values.

A critical point with a real value will be called a vertex. The cyclic order of critical values generates a cyclic enumeration of the vertices (or pair of vertices in the case of non-orientable gardens). Observe that vertices lie on the union of the boundaries of faces and they divide the boundary of each face into curve segments called edges. The restriction of $f$ to any edge $r$ has no critical points and it maps $r$ in $\overline{\mathbf{R}}$. The degree of this restricted map, i.e., the minimal cardinality of the preimage of a point of $\overline{\mathbf{R}}$ by $f$ is called the "length" of the edge $r$.

Involution $\tau$ sends the family $\Phi$ to itself interchanging the colours of all faces and preserving the rest of the structure.

Lemma 1. If a surface $P \in \Phi$ is invariant under $\tau$ (except for the colour change), then it forms a garden. The rest of components split into pairs of separated gardens.

3.2. Associated entrances, exits and alleys. By an entrance (resp. exit) we call a connected component of the inverse image $f^{-1}\left(\mathbf{C}_{+} \backslash U\right)\left(\right.$ resp. $\left.f^{-1}\left(\mathbf{C}_{-} \backslash U\right)\right)$. The weight of an entrance (resp. exit) is the genus of this connected component. Connected component of the inverse image $f^{-1}(\partial U)$ is a contour separating an entrance or an exit from a face of a garden or a separated garden of the same colour. We assign to this connected component its alley (i.e., a path) connecting the entrance/exit and the face which it separates. 
The next statement is obvious.

Theorem 2. Gardens, separated gardens, and alleys of a generic meromorphic function $f$ form a park $\mathcal{P}(f)$ which we call the park of $f$. This park is determined uniquely up to an isomorphism.

Example 2. To illustrate the two types of separate gardens, we give an example of a generic real meromorphic function and its associated park. Take a generic meromorphic function of degree four on $(T, \tau)$, where $T$ is a torus and $\tau$ is an anticonformal involution of $T$ without fixed points (i.e., $T /\langle\tau\rangle$ is a Klein bottle). Observe that such a meromorphic function has 8 critical values. Its park consists of:

(i) two separated gardens: one oriented $\left(\mathcal{G}_{0}^{\text {or }} \cup \mathcal{G}_{1}^{\text {or }}, \sigma\right)$ and one non-oriented $\left(\mathcal{G}_{0}^{\text {nor }} \cup\right.$ $\left.\mathcal{G}_{1}^{\text {nor }}, \tau\right)$

(ii) two entrances $N_{1}, N_{2}$ and two exits $X_{1}, X_{2}$, all four entrances/exits being of genus 0.

(iii) Denote by $B \mathcal{G}_{i}^{\text {or }}, B \mathcal{G}_{i}^{\text {nor }}$ and $W \mathcal{G}_{i}^{\text {or }}, W \mathcal{G}_{i}^{\text {nor }}$ the black and the white faces of the separated gardens respectively. There are three alleys from $N_{1}$ to $W \mathcal{G}_{0}^{\text {nor }}$, $W \mathcal{G}_{1}^{\text {nor }}$ and $W \mathcal{G}_{0}^{\text {or }}$ and three alleys from $X_{1}$ to $B \mathcal{G}_{0}^{\text {nor }}, B \mathcal{G}_{1}^{\text {nor }}$ and $B \mathcal{G}_{1}^{\text {or }}$, one alley from $X_{2}$ to $B \mathcal{G}_{0}^{o r}$ and one alley from $N_{2}$ to $W \mathcal{G}_{1}^{\text {or }}$.

The involution of the park sends $N_{i}$ to $X_{i}$ and

$$
\left(W \mathcal{G}_{i}^{\text {or }}, B \mathcal{G}_{i}^{\text {or }}, W \mathcal{G}_{i}^{\text {nor }}, B \mathcal{G}_{i}^{\text {nor }}\right) \rightarrow\left(B \mathcal{G}_{1-i}^{\text {or }}, W \mathcal{G}_{1-i}^{\text {or }}, B \mathcal{G}_{1-i}^{\text {nor }}, W \mathcal{G}_{1-i}^{\text {nor }}\right) .
$$

(See Figure 7.)

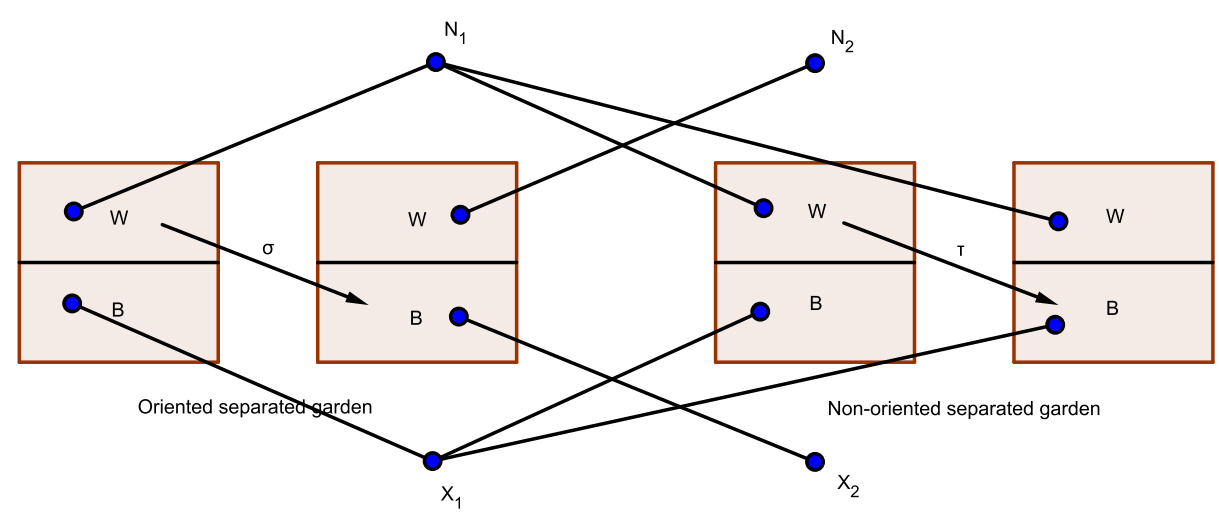

Figure 7. A park with two separated gardens.

Example 3. Consider a generic rational function of degree 3. There is a unique topological type of such functions with four critical values. Let us describe some topological types of real meromorphic functions in the Hurwitz space $H_{0,3}$. There are three possibilities. Namely, either all the critical values lie on the real cycle, or only two critical values are on the real cycle, or, finally, there are no real critical values at all.

A park for a generic real meromorphic function with four real critical values consists of a garden which is a sphere with two conjugate semi-gardens being disks with two chords. There are six faces (three white and three black), each one with an alley to an entrance/exit of genus 0 (see Figure 8).

A park of generic real meromorphic functions with two real critical values is as follows. Its semi-garden consists of a disk and a chord joining two points on the boundary. The corresponding orientable surface of the orientable garden is a sphere. 
There is an alley in each face (there are four faces) ending at an entrance/exit of genus 0 . The lengths of the three edges of each semi-garden are 0,0 and 1 .

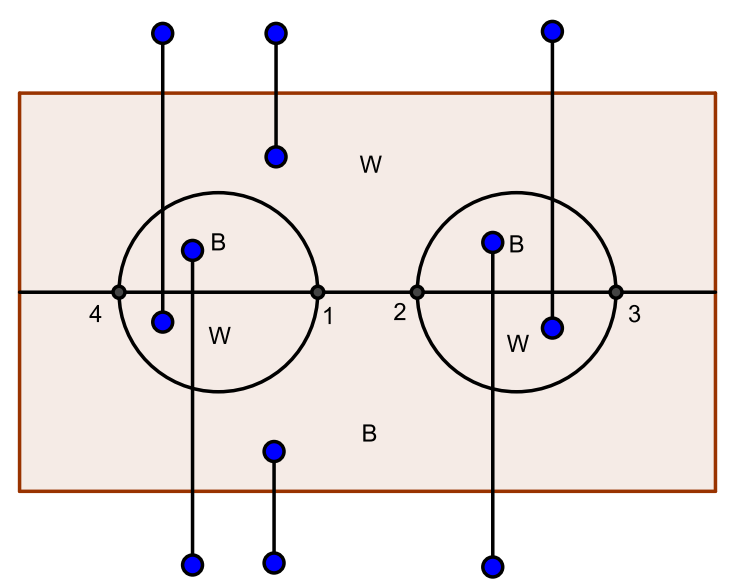

All weights and lengths are equal to 0

Figure 8. Example of a generic real rational function of degree three.

Finally, a park of a generic real meromorphic function without real critical values has a garden which is the elementary orientable garden with length 3 of its side. It has one entrance/exit, each one of genus 0 , joined by an alley with the corresponding white/black face. A different park for this case may be constructed with one elementary orientable garden, one oriented separated garden and four entrances/exits, all of them of genus 0 . The edges are three contours of length 1 .

Example 4. The park in Figure 6 corresponds to generic real meromorphic functions from Riemann surfaces of genus 13, with 10 real critical values. The orientation reversing involution has two Jordan closed curves as its fixed-point set and the quotient surface is non-orientable.

\section{Real meromorphic function corresponding to a park}

The main goal of this section is to prove the following result.

Theorem 3. For any park $\mathcal{P}$, there exists a generic real meromorphic function $f$ whose park $\mathcal{P}(f)$ is isomorphic to $\mathcal{P}$.

Proof. For any finite branched covering $f: P \rightarrow \overline{\mathbf{C}}$ of the Riemann sphere $\overline{\mathbf{C}}$ by an orientable closed 2-manifold $P$, there exists a unique holomorphic structure on $P$ such that $f$ becomes a meromorphic function, see [7]. Analogously, for any orientation-reversing involution $\tau: P \rightarrow P$ of an orientable closed 2-manifold $P$ and a finite branched covering $f: P \rightarrow \overline{\mathbf{C}}$ such that $f(\tau(p))=\bar{f}(p)$ for any $p \in P$, there exists a unique complex structure on $P$ which makes $f$ a real meromorphic function, see $[10,11]$. Thus to obtain a generic real meromorphic function realizing a given park $\mathcal{P}$, it suffices to construct a topological model of this function.

We provide such construction for parks with orientable gardens. The case for non-orientable gardens is similar.

We start with a topological model of a function realizing a garden with $n$ vertices. Consider the Riemann sphere with $n$ points on its real cycle with cyclic enumeration. The real cycle divides the Riemann sphere into white and black disks. By definition, a garden is a 2-dimensional surface split into simply-connected white and black faces. 
The boundary of each face is split by labelled vertices into edges of a given integer "length". We will construct a branched covering, which maps any face to the disk of the same colour and has at most one critical point inside each face. To obtain such a covering, it suffices to determine the mapping of the boundary of each face to the real cycle $\overline{\mathbf{R}}$. (This mapping of the boundary is uniquely determined by the coincidence of the enumerations of the vertices of the face with the cyclic enumeration of points on $\mathbf{R}$ together with the coincidence of the "length" of every edge with the degree of the mapping on this edge.)

Mappings of elementary and separated gardens are constructed similarly. By the degree of a face we call the degree of the resulting mapping of this face to a disk. The latter degree will be called the degree of the alley related to the face under consideration. This degree may be read from the park as the sum of the lengths of the edges of the face where the alley ends.

Now consider a neighbourhood of the real cycle $\overline{\mathbf{R}} \subset U \subset \overline{\mathbf{C}}$ symmetric with respect to the complex conjugation and such that it contains only real critical values of the constructed map. Its complement $\mathbf{C} \backslash U$ splits into the white and black disks $D_{+}$and $D_{-}$. The preimage of $U$ with respect to the constructed branched covering consists of gardens such that every face has exactly one hole. We call them gardens with boundary.

Now we shall construct maps corresponding to the entrances and alleys starting at them. Associate to an entrance its entrance surface whose genus equals to the weight of the entrance and the number of holes equals to the number of alleys. Consider a generic covering of this surface over $D_{+}$which maps the boundary components corresponding to the alleys to $\partial D_{+}$in such a way that the degree of the (unbranched) covering equals the degree of the alley. The number of critical points of such a covering (the latter number being determined by the Riemann-Hurwitz formula) is called the index of the entrance.

The boundary points of gardens with boundary and the boundary points of entrance surfaces are called related if their images on $\partial D_{+}$with respect to constructed coverings coincide. Now glue together the boundary components of the alleys with the corresponding boundary components of the gardens with boundary by homeomorphisms which identify related points. Using the involution, we do the same with the exit surface.

As a result, we obtain a branched covering with an orientation-reversing involution whose corresponding real meromorphic function has a park isomorphic to $\mathcal{P}$.

\section{Parks as topological types of generic real meromorphic functions}

We now settle the following statement.

Theorem 4. Two generic real meromorphic functions with isomorphic parks are topologically equivalent. The set $H(\mathcal{P})$ of all generic real meromorphic functions with a given (up to isomorphism) park $\mathcal{P}$ is a single connected component in the space of generic meromorphic functions. In other words, in the space $\mathbf{R} H^{0}$ of generic real meromorphic functions there exists a deformation transforming one generic real meromorphic function with a given park $\mathcal{P}$ to any other generic real meromorphic function with the same park.

Proof. We say that two generic real meromorphic functions are related if it is possible to deform one to the other by means of a continuous change of real critical values and pairs of complex conjugated non-real critical values. A real meromorphic 
function defined by a disjoint union of gardens and separated gardens is called simple. Construction in $\S 4$ associates to any real meromorphic function $f$ a related class of simple real meromorphic functions defined by the gardens and the separated gardens of the park of $f$. Moreover, this related class is the same for two given functions if and only if they have parks with isomorphic systems of gardens and separated gardens.

A system of entrances and its alleys of a generic real meromorphic function determines a set of ramified coverings with simple critical values $\varphi_{i}: P_{i} \rightarrow D$ of degrees $d_{i}$, where $P_{i}$ is a Riemann surface of genus $g_{i}$ with $k_{i}$ holes and $D$ is a disk. Consider the degrees $d_{i}^{1}, \ldots, d_{i}^{k_{i}}$ of $\left.\varphi_{i}\right|_{\partial P_{i}}: \partial P_{i} \rightarrow \partial D$ on components $c_{j} \subset \partial P_{i}$. It follows from $[8,10]$ and Chapter 3 of [11] that any other covering $\varphi_{i}^{\prime}: P_{i}^{\prime} \rightarrow D$ is obtained from $\varphi_{i}$ by a continuous change of critical values if the topological types $\left(g_{i}, k_{i}\right)$ and $\left(g_{i}^{\prime}, k_{i}^{\prime}\right)$ respectively of $P_{i}$ and $P_{i}^{\prime}$ are the same, the degrees $d_{i}$ and $d_{i}^{\prime}$ of $\varphi_{i}$ and $\varphi_{i}^{\prime}$ are the same, and the degrees $d_{i}^{1}, \ldots, d_{i}^{k_{i}}$ and $d^{\prime 1_{i}}, \ldots, d_{i}^{\prime k_{i}}$ on the boundary components are the same.

The park of a generic real function defines the topological type $\left\{\left(g_{i}, k_{i}\right)\right\}$ of the system of entrances and its alleys and the corresponding degrees $d_{i}^{1}, \ldots, d_{i}^{k_{i}}$. Thus for functions with isomorphic parks, there exists a continuous change of critical values deforming one system of entrances and its alleys to the other. The anti-holomorphic involution generates a continuous change of critical values deforming one system of exits and its alleys to the other. Together with the change of real critical values which we considered above, this generates a continuous change of critical values transforming one real function to the other.

\section{Hurwitz numbers}

A Hurwitz number is, by definition, a weighted number of equivalence classes of coverings with fixed critical values. Generic real meromorphic functions belong to different equivalence classes if and only if they have different topological types (i.e., non-equivalent parks). Thus it is enough to find a Hurwitz number for any given topological type.

Consider the set $H(\mathcal{P})$ of all generic real meromorphic functions with a given (up to an isomorphism) park $\mathcal{P}$. In $\S 4$ we constructed a decomposition of a generic real meromorphic function into a real function defined on the system of gardens and separated gardens and a function defined on surfaces generated by entrances and exits with alleys. Thus the Hurwitz number for $H(\mathcal{P})$ is obtained by multiplication of the Hurwitz number for the function corresponding to the gardens and separated gardens by the Hurwitz number for the function defined on the surfaces generated by the entrances and its alleys. (Notice that the function defined on the surfaces generated by the entrances and its alleys determines the function defined on the surfaces generated by the exits and its alleys.)

The main construction of $\S 4$ demonstrates that the Hurwitz number for the functions corresponding to the gardens and separated gardens equals 1 . This fact means that in order to calculate the total Hurwitz number, it suffices to determine the Hurwitz number for the function defined on the system of surfaces generated by the entrances and its alleys. This function, in its turn, is a union of functions with simple critical values on surfaces $P_{i}$ of topological types $\left\{\left(g_{i}, k_{i}\right): i=1, \ldots, n\right\}$ and degrees $\left\{d_{i}^{j}: j=1, \ldots, k_{i}\right\}$ on boundary components, see $\S 5$. 
Collapsing the boundary components of $P_{i}$ and the boundary of the image disk to a point, we obtain a map of the closed surface $\bar{P}_{i}$ to the Riemann sphere which have only one non-simple critical value. The number of its simple critical values is calculated by the Riemann-Hurwitz formula and is equal to

$$
b_{i}=2 g_{i}-2+k_{i}+\sum_{j=1}^{k_{i}} d_{i}^{j} .
$$

The Hurwitz number $H_{g_{i}}\left(d_{i}^{1}, \ldots, d_{i}^{k_{i}}\right)$ for such a map is equal to the Hurwitz number corresponding to the coverings of $P_{i}$ over a disk and is called a single Hurwitz number. These numbers, appearing in different areas of mathematics from moduli spaces to integrable systems, are well studied.

Now the Hurwitz number for the topological type given by all entrances and its alleys equals

$$
\frac{\left(b_{1}+\cdots+b_{n}\right) !}{b_{1} ! \ldots b_{k} !} \prod_{i=1}^{n} H_{g_{i}}\left(d_{i}^{1}, \ldots, d_{i}^{k_{i}}\right) .
$$

As we explained above, this number coincides with the Hurwitz number for generic real meromorphic functions of type $\mathcal{P}$.

Example 5. Consider the park $\mathcal{P}$ in Figure 2 where all the lengths of sides are 0 . There is only one entrance of genus 3 with two boundary components of degree 1 . The number $b$ is 8 and the Hurwitz number for the topological type of generic real meromorphic functions given by $\mathcal{P}$ is

$$
H(\mathcal{P})=\frac{8 !}{8 !} H_{3}(1,1)
$$

\section{Parks and monodromy of coverings}

The traditional way to describe coverings and meromorphic functions is by using their monodromy. In the case of a degree $d$ generic meromorphic function $f: P \rightarrow \overline{\mathbf{C}}$, the monodromy is a representation $\omega: \pi_{1}\left(\overline{\mathbf{C}} \backslash\left\{z_{1}, \ldots, z_{n}\right\}\right) \rightarrow \mathrm{Sym}_{d}$, where $\left\{z_{1}, \ldots, z_{n}\right\}$ is the set of critical values and $\operatorname{Sym}_{d}$ is the symmetric group of permutations of $d$ elements. For a generic meromorphic function, $\omega$ sends each based small loop encircling a singular value to a transposition. The monodromy tells us how the elements of $\pi_{1}\left(\overline{\mathbf{C}} \backslash\left\{z_{1}, \ldots, z_{n}\right\}\right)$ lift to the surface $P$ and $\omega^{-1}(\operatorname{Stab}(1))$ is isomorphic to the group $\pi_{1}\left(P \backslash f^{-1}\left\{z_{1}, \ldots, z_{n}\right\}\right)$. Two monodromy representations correspond to topologically equivalent meromorphic functions if and only if there exists an automorphism of $\pi_{1}\left(\overline{\mathbf{C}} \backslash\left\{z_{1}, \ldots, z_{n}\right\}\right.$ ) (given by an orientation-preserving homeomorphism) and an automorphism of $\mathrm{Sym}_{d}$ sending one representation to the other. In this way, it is possible to prove that there exists a unique topological type of degree $d$ generic meromorphic functions from Riemann surfaces of a fixed genus (see [2] and [5]).

The degree $d$ generic real meromorphic functions may be described by a degree $d$ orbifold covering $\hat{f}: \widehat{P} \rightarrow D_{t, \bar{s}}$ where $D_{t, \bar{s}}$ is an orbifold with the topological type of a disc, $t$ conic points of order two and $s$ corner points of angle $\pi / 2$. If $P$ is the complex double of $P$, the covering $\widehat{f}$ provides $f: P \rightarrow \overline{\mathbf{C}}$ which is a generic meromorphic function with $2 t+s=n$ critical values, $s$ of them real. These coverings are given by a monodromy representation

$$
\varpi: \pi_{1} O\left(D_{t, \bar{s}}\right) \rightarrow \operatorname{Sym}_{d}=\operatorname{Sym}\{1, \ldots, d\} .
$$


The group $\pi_{1} O\left(D_{t, \bar{s}}\right)$ has a presentation:

$$
\left\langle x_{1}, \ldots, x_{t}, e, c_{1}, \ldots, c_{s+1}: x_{i}^{2}=c_{i}^{2}=\left(c_{i} c_{i+1}\right)^{2}=1 ; x_{1} \ldots x_{t} e=1 ; c_{1}=e c_{t+1} e^{-1}\right\rangle \text {. }
$$

Since $\varpi$ is given by a generic real meromorphic function, it satisfies some specific properties:

1. the permutations $\varpi\left(x_{i}\right)$ are transpositions;

2. the permutations $\varpi\left(c_{i} c_{i+1 \bmod s}\right)$ are transpositions or products of two (disjoint) transpositions;

3. every $\varpi\left(c_{i}\right)$ is an element of order two.

The entrances of the park are given by the orbits of the subgroup generated by

$$
\left\langle\varpi\left(x_{1}\right), \ldots, \varpi\left(x_{t}\right)\right\rangle .
$$

The white faces and the corresponding alleys are in $1-1$ correspondence with the orbits of the element $\varpi(e)$. The face corresponding to an orbit of $\varpi(e)$ is joined with the entrance which is the orbit of $\left\langle\varpi\left(x_{1}\right), \ldots, \varpi\left(x_{t}\right)\right\rangle$ containing it.

The exits, black faces and corresponding alleys are obtained using the orbits of

$$
\left\langle\varpi\left(c_{1} x_{1} c_{1}\right), \ldots, \varpi\left(c_{1} x_{t} c_{1}\right)\right\rangle
$$

and the cycles of $\varpi\left(c_{1} e c_{1}\right)$.

Example 6. We can apply Theorem 4 to check if two generic real meromorphic functions given by its monodromies have or not the same topological type. For example, let $f_{1}: P_{1} \rightarrow \overline{\mathbf{C}}$ and $f_{2}: P_{2} \rightarrow \overline{\mathbf{C}}$ be two generic real meromorphic functions of degree $d$ without real critical values from two surfaces of a fixed genus $g \geq 2$. Let

$$
\varpi_{i}: \pi_{1} O\left(D_{t, \overline{0}}\right) \rightarrow \operatorname{Sym}\{1, \ldots, d\}, i=1,2,
$$

be the monodromy representations of $\widehat{f}_{1}$ and $\widehat{f}_{2}$ respectively. Assume that $\varpi_{1}(e)$ and $\varpi_{2}(e)$ are $d$-cycles, and that the real involutions on $P_{1}$ and $P_{2}$ are separating. The corresponding garden of each function is an elementary garden with length $d$ on its edge and there is only one entrance and one exit in each park. Hence the parks are equivalent, $\left(P_{1}, f_{1}\right)$ and $\left(P_{2}, f_{2}\right)$ are topologically equivalent.

\section{References}

[1] Alling, N. L., and N. Greenleaf: Foundation of the theory of Klein surfaces. - Lecture Notes in Math. 219, 1971.

[2] Clebsch, A.: Zur Theory der algebraischen Funktionen. - Math. Ann. 29, 1887, 171-186.

[3] Costa, A.F., M. Izquierdo, and G. Riera: One-dimensional Hurwitz spaces, modular curves, and real forms of Belyi meromorphic functions. - Int. J. Math. Sci. 2008, Art. ID 609425, 2008, 1-18.

[4] Costa, A.F., and G. Riera: One parameter families of Riemann surfaces of genus two. Glasg. Math. J. 43, 2001, 255-268.

[5] Eisenbud, D., N. Elkies, J. Harris, and R. Speiser: On the Hurwitz scheme and its monodromy. - Compos. Math. 77, 1991, 95-117.

[6] Hurwitz, A.: Über die Anzahl Riemannschen Flächen mit gegebenen Verzweigungspunkten. - Math. Ann. 55, 1902, 53-66.

[7] KeréKJartó, B. V.: Vorlesungen über Topologie. I. Flächentopologie. - Grundlehren Math. Wiss. 8, Springer-Verlag, Berlin, 1923.

[8] Natanzon, S. M.: Spaces of real meromorphic functions on real algebraic curves. - Soviet Math. Dokl. 30, 1984, 724-726. 
[9] Natanzon, S. M.: Real meromorphic functions on real algebraic curves. - Soviet Math. Dokl. $36,1987,425-426$.

[10] Natanzon, S. M.: Topology of 2-dimensional coverings and meromorphic functions on real and complex algebraic curves. - Selecta Math. Sovietica 12, 1993, 251-291.

[11] Natanzon, S. M.: Moduli of Riemann surfaces, real algebraic curves, and their superanalogs. - Transl. Math. Monogr. 225, Amer. Math. Soc., Providence, RI, 2004.

[12] Natanzon, S. M., and S. V. Shadrin: Topological classification of real meromorphic functions in general position on separating curves. - Dokl. Akad. Nauk 388, 2003, 449-451.

[13] Natanzon, S. M., B. Shapiro, and A. Vainshtein: Topological classification of generic real rational functions. - J. Knot Theory Ramifications 11:7, 2002, 1063-1075.

[14] VAKIL, R.: Genus 0 and 1 Hurwitz numbers: recursions, formulas, and graph-theoretic interpretations. - Trans. Amer. Math. Soc. 353:10, 2001, 4025-4038.

Received 17 January 2017 • Revised received 21 April 2017 • Accepted 29 August 2017 